\title{
IDENTIFICATION AND ANALYSIS OF INFRARED LINES OF WC STARS
}

\author{
P.R.J. EENENS ${ }^{1}$, P.M. WILLIAMS ${ }^{2}$ and R. WADE ${ }^{3}$ \\ 1 Department of Astronomy, University of Edinburgh, Blackford Hill, \\ Edinburgh EH9 $3 \mathrm{HJ}$, United Kingdom \\ 2 Royal Observatory, Blackford Hill, Edinburgh EH9 $3 H J$, United Kingdom \\ 3 Joint Astronomy Centre, 665 Komohana St, Hilo, HI 96720, USA
}

\begin{abstract}
We present $1-3.4 \mu \mathrm{m}$ spectra of six Wolf-Rayet stars: WR 146 (WC4), WR 111 (WC5), WR 86 (WC7), WR 140 (WC7+04), WR 135 (WC8) and WR 88 (WC9). Examination of the relative strengths of the emission lines has enabled us to make over 20 new line identifications. Recombination analyses of the carbon and helium lines yield $\mathrm{C} / \mathrm{He}$ abundance ratios much in excess of the solar value and correlated with spectral subtype.
\end{abstract}

The spectra were observed at resolutions of $300-600$ with the cooled grating spectrometer (CGS2) on the United Kingdom Infrared Telescope (UKIRT). The spectra of C III, C IV, He I and He II are well represented. About 20 lines are identified for the first time, most conspicuously the C IV $4 \mathrm{p}-4 \mathrm{~s}$ doublet at $1.435 \mu \mathrm{m}$, observed previously but not identified. We also identified fine structure lines of $\mathrm{C}$ III and (more tentatively) of oxygen (O II, O III, O V); and satellite lines of the C III 7-6 and C IV 10-9, 9-8 and 8-7 arrays. Satellite and fine stucture lines are specially useful to help identify the contributors to the strong hydrogenic transition arrays. Even a few helium lines had not been reported before: He I 5p-3d (at 1.299 $\mu \mathrm{m}$ ), He II 17-7 (at $1.344 \mu \mathrm{m}$ ) and He II 16-7 (at 1.381 $\mu \mathrm{m}$ ). The 2p-2s He I lines show P Cygni profiles, the absorption component of the triplet $(1.083 \mu \mathrm{m})$ being stronger in earlier subtypes and the singlet $(2.058 \mu \mathrm{m})$ in later subtypes. The line ratios correlate with spectral subtype but the spectrum of WR 146 seems anomalous: the C IV lines indicate WC7 or later while the He II lines indicate WC4.

We followed the recombination method of Hummer, Barlow and Storey (IAU 99, p.277, 1982) to derive relative abundances for icns of $\mathrm{He}$ and $\mathrm{C}$. The analysis shows $\mathrm{C} / \mathrm{He}$ abundances much in excess of the solar value, as well as a decrease of the $\mathrm{C} / \mathrm{He}$ ratio with WC subtype, thus qualitatively supporting the predictions of evolutionary models that the later WC subtypes evolve into the earlier. 